\title{
ESTUDIO SOBRE EL CRECIMIENTO DEL PAUTAJE PUBLICITARIO EN LOS CINES DE GUAYAQUIL ECUADOR.
}

\section{STUDY ON THE DEVELOPMENT OF ADVERTISING IN THE CINEMAS \\ OF GUAYAQUIL, ECUADOR.}

\author{
Edgar Salas $^{1}$
}

\begin{abstract}
Palabras clave: Resumen
Preferencias del El presente trabajo de investigación se enfoca en analizar el consumidor, mercado del cine ecuatoriano, con mayor énfasis en las Ecuador, cine. ciudades de Guayaquil, y Samborondón, su crecimiento y las preferencias del consumidor. El cine como tal tiene una historia a través de los años que ha logrado un gran impacto hacia la atracción del público en masas en un solo establecimiento, es así como en cuestión de años grandes marcas han encontrado efectos positivos en canales de publicidad como las pantallas del cine. A lo largo de la investigación se van a exponer los beneficios, empresas que publicitan, antecedentes relacionados con el cine en un ambiente tanto internacional como nacional. Para poder evaluar las variables se realizaron 200 encuestas dirigidas a los consumidores. Fue así como se determinó que dentro de las 2 cadenas de cine existentes en Ecuador, Supercines supera las preferencias del consumidor y es reconocido por su disponibilidad de horarios. De esta manera, se demostró que existen un sin número de preferencias del consumidor y su perspectiva en cuanto a la publicidad minutos antes de sus películas. Finalmente, la publicidad dentro de los cines alcanza un nivel importante de recordación de marca y sigue siendo un buen elemento para grandes empresas.
\end{abstract}

Códigos JEL: M37

\footnotetext{
${ }^{1}$ Universidad de Especialidades Espíritu Santo, (Ecuador).

E-mail: esalas@uess.edu.ec
} 
Keywords:

Consumer

preferences,

Ecuador, movie

theater

\section{Abstract}

This research work focuses on analyzing the Ecuadorian film market, with greater emphasis on the cities of Guayaquil, and Samborondón, its growth and consumer preferences. The cinema as such has a history through the years that has achieved a great impact towards the attraction of the mass public in a single establishment, this is how in a matter of years great brands have found positive effects in advertising channels such as screens of the cinema. Throughout the investigation the benefits will be exposed, companies that advertise, background related to the cinema in an international and national environment. In order to evaluate the variables, 200 consumer surveys were conducted. It was thus determined that within the 2 existing film chains in Ecuador, Supercines exceeds consumer preferences and is recognized for its availability of schedules. In this way, it was shown that there are a number of consumer preferences and their perspective in terms of advertising minutes before their movies. Finally, the advertising within the cinemas reaches an important level of brand recall and remains a good element for big companies.

JEL Code: M37 


\section{INTRODUCCIÓN}

El cine ha sido uno de los mayores avances en la industria artística. Desde 1924, las producciones cinematográficas nacen en Ecuador, convirtiéndose en una marca definitiva en el desarrollo del país; a pesar de que esta transición no ha logrado el mismo reconocimiento que industrias de otros países en Latinoamérica. Unos breves datos, coadyuvan a entender desde cuándo tuvo su origen el cine como un medio para la publicidad.

El cine publicitario lo inventaron los Lumière, que no sólo introdujeron en su catálogo cintas de contenido publicitario, sino que, además, se dedicaron a promover el capitalismo al exhibir en sus pantallas unas formas de vida burguesas que parecían perfectamente imitables por la clase obrera. No debemos olvidar que el cine, en sus primeros años de existencia, era un espectáculo para los ricos. En muy poco tiempo el cine se convirtió en un espectáculo de masas. La opulencia de aquellas escenas, la exhibición de los lugares de vacaciones de los ricos, el retrato en movimiento de sus actividades de ocio, de su forma de vestir, de su manera de comportarse en sociedad, invitaba a los trabajadores a desear una vida desahogada que podía lograrse perfectamente con el consumo de objetos. Desde un punto de vista estrictamente publicitario, los Lumière introdujeron las marcas comerciales en su catálogo cuando, en el año 1896, Alexander Promio, filmó en Suiza la película Les Laveuses, en la que el protagonista de la escena era el jabón Sunlight, fabricado por la casa Lever (Sánchez, 2010).

En ese sentido, el cine como tal, ya no es simplemente una producción de filmes y largometrajes. En la actualidad, esta sociedad es un proceso complejo e integral de intervención estética, política y cultural. Bajo el escenario de una organización estructural, en lo que respecta a lo comercial, creativa-profesional de las producciones, existe una escasez de empresas que sirvan al sector de una capacidad comercial y de posicionamiento en los mercados.
En Europa, específicamente en España, el medio cine, es el medio que menos ha evolucionado en términos de control de actividad en publicidad, incluso, este medio se incorporó más tarde al control de la actividad publicitaria, sin embargo, existen empresas que controlan la actividad en el cine, como lo es la empresa Arce Media, quien tiene bajo su coyuntura, toda la actividad proyectada en las salas comercializadas por los exclusivistas que colaboran con estas fuentes, además de registrar el ámbito de la publicidad, registra la publicidad local como la nacional y el seguimiento (aquella asociada a una película). La información que se le asocia a cada inserción publicitaria es al siguiente: Información básica sobre el soporte: fecha de proyección, provincia de la sala, el exclusivista, topos de publicidad o ámbito de la publicidad. A esto se suma, una asignación de sector o grupo, un producto, una marca, un modelo, un submodelo y un anunciante (Sánchez, 2010).

En lo que respecta a Ecuador, el 22 de mayo de 1968 se crea la Asociación Ecuatoriana de Agencias de Publicidad (AEAP) (2014), cuyos objetivos principales eran profesionalizar la actividad publicitaria y generar una relación sólida con los medios de comunicación y anunciantes, en beneficio de los consumidores.

Con el paso de los años, se ha venido estableciendo nuevas normativas que contemplan restricciones que impone la Ley Orgánica de Comunicación, ya que no son proporcionales ni constituyen el único medio para satisfacer el objetivo que se pretende. Además, existe una vulneración a la libertad de contratación pues al determinar que la publicidad tiene que ser producida en el Ecuador, no se permite al anunciante ecuatoriano que contrate a una empresa extranjera para la producción de su publicidad, al igual que un extranjero que desee publicitar su marca en el Ecuador debe forzosamente contratar con una agencia de publicidad ecuatoriana (Muñoz, 2015). 
Por su parte, un estudio realizado en Guayaquil, capital comercial de Ecuador, acerca de los canales de promoción del producto en el cine, se concluyó que existe una falta de promoción por canales adecuados y no contar con elementos de producción atractivos. Otro resultado importante es que la publicidad por este medio no es concebida de manera satisfactoria, ya que, en el momento de la selección del medio óptimo para la distribución de las películas es que existe un alto número de personas que prefieren el internet a las salas de cine. El 38\% de los encuestados consume más cine por internet que asistiendo a salas de exhibición y, el consumo en salas de cine está en segundo lugar $(22 \%)$ y en tercero el DVD $(20 \%)$.

Otro rasgo importante de comportamiento del espectador revelado en la investigación, en relación con la importancia de la publicidad como criterio de selección, es el medio a través del cual reciben información sobre los estrenos cinematográficos. En ambas ciudades y rangos de edad, el estudio señala que el (43\%) prefiere las redes sociales o el internet como la fuente principal de información. Esto sugiere que la industria del cine como la publicidad, podrían considerar invertir más en campañas a desarrollarse, en redes sociales, que en medios tradicionales al momento de promocionar sus películas y productos (García, 2017).

En lo que se refiere a la producción, distribución, consumo y recepción del cine en el Ecuador, existe un notable crecimiento de la producción cinematográfica nacional en un $300 \%$. El estudio consultado revela que existen problemas dentro de la etapa de circulación, específicamente con la distribución y su relación con la comercialización debido a factores internos como externos; estos problemas han generado que no se desarrolle la figura del distribuidor. Un claro ejemplo, es el de Ecuador, con respecto a un estudio de audiencias el número de espectadores en el 2013 no rebasó las 55.000 personas. Por otro lado, las películas "La Tigra" 1990 "Que tal Lejos" 2006 lograron cifras superiores a los 250.000 (El Comercio, 2013).
De acuerdo con García (2015) algunos de los productores justifican la no realización de un plan de negocios o estudio de mercado, en el hecho de que no existe un verdadero registro en términos de cifras de distribución por película nacional en el Ecuador. Sin embargo, se pueden encontrar datos generales en base a los cuales hacer estimaciones y proyecciones para construir estudios de mercado. En el cine se pueden obtener cifras generales de taquilla, en publicaciones y periódicos se pueden obtener datos de presupuestos de películas dados por los mismos realizadores. Por ende, sí podría ser factible tener una visión general del mercado que permita diseñar un plan de producción viable de acuerdo a la realidad del país, aunque no con el detalle ideal al que aspiran los productores.

A manera de comparación entre Europa y Ecuador, Sánchez (2010, pp. 87) expresa que la publicidad audiovisual en Europa se destaca en las cintas que Bori y Gardó denominaron composiciones, que no son otra cosa que películas publicitarias de corta duración realizadas con fines estrictamente comerciales: lo que hoy en día conocemos como «anuncios». Este tipo de composición fue el que consiguió imponer su lenguaje en el ámbito de la publicidad cinematográfica, que se desarrolló prioritariamente a partir de pequeñas historias realizadas expresamente para dar a conocer una marca o producto.

En lo que respecta al método de publicidad ecuatoriano, no existe hasta el momento, empresas encargadas o que brinden servicios de distribución de las realizaciones cinematográficas, esta es una tarea donde los propios directores y/o productores facilitan sus contactos para realizar negociaciones en los canales que consideren los más adecuados, por lo que, si no existe la figura del consumidor del medio cinematográfico, no podría existir una audiencia que conozca el producto publicitado por este canal.

En consecuencia, de ello, por varios años, muchas compañías han buscado publicitarse mediante estos largometrajes. Sin embargo, existen algunas marcas que no consideran el hecho de que su nombre sea 
utilizado o visualizado a lo largo de la cinta; más bien, prefieren ser utilizados como anuncios publicitarios durante el tiempo de proyección antes de una película en una sala de cine.

Este trabajo tiene como objetivo determinar cómel crecimiento de la publicidad en los cines. También, busca mostrar cuáles han sido las marcas que suelen pautar su transmisión en las salas. Además, intenta explicar por qué la mayoría de los anuncios publicitarios transmitidos en las salas de los cines a nivel nacional son internacionales. $\mathrm{Y}$, mediante resultados de encuestas, informar el nivel de aceptación de las personas ante estos comerciales transmitidos antes de las películas en los cines.

\section{MARCO TEÓRICO}

Para poder desarrollar un mejor análisis del tema a tratar acerca del desarrollo de la publicidad en los cines ecuatorianos, se han seleccionado ciertos términos y palabras específicas, que facilitaran el entendimiento del tema.

Según (Pérez Porto \& Merino, 2018), se entiende como cine, a aquel registro y la exhibición de imágenes en movimiento sobre una pantalla.

Así también, según (¿Qué es Cine?, 2015), se denomina "cine", al espacio físico en donde se encuentra el local o sala equipada, donde se exhiben películas para el público sentados en butacas.

Según (Conocimientos, Marketing, Ventas, 2012), el término "mercado", también puede entenderse como marketing, es el lugar en donde coinciden para hacer sus transacciones los compradores, quienes serían la demanda, y los vendedores, que son la oferta.

La publicidad se define como un conjunto de estrategias orientadas a dar a conocer diversos productos y servicios a la sociedad; aunque, no esté empleo no solo busca que el público sepa que existen, sino también de otorgar cierta reputación a lo que se ofrece (La Publicidad, 2019). Sin utilizar esta comunicación patrocinada, sería muy complejo posicionarse en la mente de los consumidores como algo de buena calidad.

La industria cinematográfica ha realizado cambios extraordinarios en nuestro país. Los cines son considerados un consumo masivo, en los cuáles, la publicidad se encuentra presente ejecutando un rol fundamental.

Con todas las particularidades que presenta esta industria, se pueden diferenciar tres tipos de cines: el cine comercial (que apunta a captar la atención de un público masivo), el cine de autor (centrado en las decisiones del director) y el cine experimental (con poca o ninguna estructura narrativa).

Hoy en día, la publicidad suele ser orientada entre lo tradicional y lo digital. Los anuncios publicitarios tienen un papel importante: sirven para divulgar productos, servicios y marcas, con el objetivo de estimular las relaciones comerciales (La Publicidad, 2019). A muchas personas no les agrada el ver los anuncios que suelen ser transmitidos en el cine. Sin embargo, diversos estudios han afirmado que este tipo de comerciales que suelen ser mostrados en la gran pantalla, resultan ser más recordados que los que se son transmitidos en televisión (Olivas, 2014).

Según un estudio publicado por el sitio web Puro Marketing (2008) el 65\% de las personas encuestadas, han recordado la asociación entre marca y productos que son promocionados antes de la proyección de las películas en el cine y donde el nivel de recuerdo asciende hasta un $90 \%$ cuando se les comenta o cuando se les pregunta por la publicidad que habían visto anteriormente.

Este tipo de comunicación pagada impacta y es mayormente recordada. Cada sala tiene una transmisión publicitaria diferente; en su mayoría, basadas en el tamaño y la oferta de las películas. Y, generalmente, detrás de estos comerciales, hay una gran producción de por medio. 
Según un reporte publicado en Diario El Comercio por (Veintimilla, 2018) a partir del año 2013, La Ley Orgánica de Comunicación establece que la publicidad que se difunda en territorio ecuatoriano a través de los medios de comunicación deberá ser producida por personas naturales o jurídicas ecuatorianas y cuya nómina para su realización y producción la constituyan al menos un $80 \%$ de personas de nacionalidad ecuatoriana o extranjeros legalmente radicados en el país. El art. 98 respalda y obliga a transmitir, en los medios nacionales, piezas publicitarias realizadas con un $80 \%$ de personal ecuatoriano o extranjeros nacionalizados.

Entonces, ¿por qué la mayoría de los anuncios publicitarios que vemos en las salas de los cines a nivel nacional, son internacionales? En nuestro país, las dos cadenas de cines más reconocidas son Supercines y Cinemark. Tanto la una como la otra, manejan un alto número de publicidad antes de sus transmisiones cinematográficas. En el sitio oficial de Supercines (2019), se puede observar algunas de las marcas que utilizan este medio para publicitarse; algunas de ellas son Chili's, Red Lobster y Carl's Jr. (marcas internacionales). Entonces, aquí se resuelve la incógnita: esto se debe a que normalmente, los cines suelen publicitar, en su mayoría, marcas que pertenezcan a las mismas compañías dueñas de las franquicias; estos son los casos de las marcas mencionadas anteriormente.

Las publicidades en cines se distinguen cuando grandes marcan buscan generar contacto con el público. Desde siempre, las personas que visitaban los cines veían sólo avances de otras películas antes de iniciar la función; pero, a medida que pasaba el tiempo, se volvió más común ver diez o más comerciales de marcas que buscan conectarse con el público. Entonces, si una persona elige ir al cine como distracción, brinda su atención a la pantalla y todo lo que proyecte.

\section{METODOLOGÍA}

\section{Diseño de investigación}

Este trabajo analiza la efectividad de la publicidad pautada en los cines desde una perspectiva mixta, relacionando indicadores objetivos con variables demográficas y de percepción de los consumidores. Siguiendo los trabajos de Wells, Burnett, \& Moriarty (1995) y Yuan (2017), se apela a los niveles de exposición y de atención como medidas del grado de efectividad publicitaria. La exposición, hace referencia al tiempo que se expone la audiencia a los anuncios publicitarios, y la atención, se entiende como la tasa de recuperación de anuncios post función (Ewing, Du Plessis, \& Foster, 2001). La exposición distingue entre grupos que son asignados por el instante en el que se visualizan las pautas publicitarias; la atención por su parte ha sido captada en la literatura a través del recuerdo o recuperación de marcas (Singh, Rothschild, \& Churchill, 1988). Ambos enfoques serán tomados en cuenta para su posterior medición.

Además, se estudia las diferencias en los niveles de efectividad publicitaria por género y edad. Luego, como aporte de este trabajo, se explora el vínculo entre; la percepción de los consumidores sobre la pertinencia de la publicidad en los cines y los niveles de recuperación de anuncios publicitarios, en respuesta al desagrado de los espectadores al observar anuncios antes de las películas, que alcanza entre el $10 \%$ y el $15 \%$ del total de la audiencia (Stanley, 1995). Adicionalmente se proporcionará un análisis descriptivo sobre los gustos y preferencias que rodean a los consumidores en las elecciones que deben realizar cuando asisten al cine.

\section{Instrumento y muestra}

Se desarrolló un cuestionario estructurado con 10 ítems, dividido en tres secciones. La sección Demográfica recoge las variables de Edad y Género; La sección de Medidas de efectividad recoge información relevante para la construcción de las medidas propuestas y La sección Criterios de preferencia extrae información útil que permitirá contextualizar el análisis.

Las variables de efectividad publicitaria fueron valoradas de la siguiente forma: para medir el nivel de Exposición a la publicidad se clasifico a los 
participantes en tres grupos, aquellos que ingresaron a la sala de cine antes de la publicidad, durante la publicidad y después de la publicidad. El nivel de Recuperación de marcas se captó por medio de una pregunta abierta, en el que solicitó a los participantes enunciar la cantidad de marcas que recuerdan haber observado en la última función a la que asistieron.

La Percepción sobre la pertinencia de la publicidad se clasificó en atractiva, indiferente y molestosa. En sintonía con los trabajos previos, se

clasifico a los participantes de acuerdo con un determinado rango de edad; aquellos con edades entre 18 y 35 años se les asigno al grupo de Adulto joven y los que presentan edades mayores a 36 años se los asigno al grupo de Adulto mayor.
Las respuestas solicitadas en el cuestionario tienen como criterio de referencia; la experiencia vivida en la última visita al cine del participante, con una extensión máxima de 7 días, contando desde el momento en que recibe el cuestionario. Los datos fueron recopilados a través de un trabajo de campo efectuado durante todo el mes de febrero del presente año. El cuestionario fue aplicado de manera física en las ciudades de Guayaquil y Samborondón, Ecuador. Se encuestaron un total de 195 personas, que representa el tamaño de muestra de este estudio, de los cuales, 91 son hombres y 104 son mujeres.

En la tabla 1 se detalla las variables capturadas con las distintas opciones disponible.

Tabla 1.

Descripción de variables.

\begin{tabular}{|c|c|c|}
\hline Sección & Variable & Opciones \\
\hline \multirow{2}{*}{ Demográfica } & Género & $\begin{array}{c}\text { Hombre } \\
\text { Mujer }\end{array}$ \\
\hline & Edad & $\begin{array}{c}\text { 18-35 (Adulto joven) } \\
>36 \text { (Adulto mayor) }\end{array}$ \\
\hline \multirow{3}{*}{$\begin{array}{l}\text { Medidas de } \\
\text { efectividad }\end{array}$} & Percepción sobre la pertinencia de la publicidad & $\begin{array}{c}\text { Atractiva } \\
\text { Indiferente } \\
\text { Molestosa }\end{array}$ \\
\hline & Exposición a la publicidad & $\begin{array}{c}\text { Antes de la publicidad } \\
\text { Durante la publicidad } \\
\text { Después de la publicidad } \\
\end{array}$ \\
\hline & Recuperación de marcas & Pregunta abierta (número de marcas recordadas) \\
\hline \multirow{5}{*}{$\begin{array}{l}\text { Criterios de } \\
\text { Preferencia }\end{array}$} & Frecuencia de visita & $\begin{array}{c}\text { Una vez por semana } \\
2 \text { a } 3 \text { veces al mes } \\
\text { Una vez al mes } \\
\text { Cada } 2 \text { meses o más }\end{array}$ \\
\hline & Motivo de preferencia & $\begin{array}{c}\text { Instalaciones } \\
\text { Películas subtituladas } \\
\text { Variedad de horarios } \\
\text { Variedad de formatos (3D) } \\
\end{array}$ \\
\hline & Método de compra & $\begin{array}{l}\text { Aplicación móvil } \\
\text { Ventanilla } \\
\text { Sitio web } \\
\end{array}$ \\
\hline & Género de película preferida & $\begin{array}{c}\text { Acción } \\
\text { Ciencia ficción } \\
\text { Comedia } \\
\text { Drama } \\
\text { Infantil } \\
\text { Romance } \\
\text { Terror } \\
\end{array}$ \\
\hline & Actividad alternativa & $\begin{array}{l}\text { Ir a un restaurante } \\
\text { Practicar un deporte } \\
\text { Ver Netflix } \\
\text { Salir de paseo } \\
\text { Ir al teatro }\end{array}$ \\
\hline
\end{tabular}




\section{Hipótesis y Procedimiento}

Las hipótesis presentadas en esta sección siguen la línea de investigación reciente en el que se evalúa el grado de efectividad del pautaje publicitario con las características demográficas de los consumidores. Las cuatro primeras hipótesis mantienen dicho enfoque. En total se plantean 5 hipótesis.

Tabla 2.

Estado de las hipótesis.

Etiqueta Hipótesis

\section{Test}

Las mujeres tienen un mayor grado de exposición a la publicidad en el cine que los hombres.
Chi-cuadrado

Chi-cuadrado

t-Student

Las mujere
hombres

Los adultos más jóvenes tienen un mayor nivel de recuperación de marcas en el cine que los adultos mayores.
t-Student

ANOVA
La hipótesis $\mathrm{H} 1$ relaciona el género de los participantes con el nivel de exposición a la publicidad, esto debido a que las mujeres presentan una posibilidad mayor de estar sentadas en el auditorio antes de la proyección de los anuncios que los hombres (Dunnett \& Hoek, 1996), por lo tanto, se espera que las mujeres tengan una mayor tasa de exposición a la publicidad en el cine. En respuesta a la hipótesis $\mathrm{H} 2$, que relación la edad con el nivel de exposición a la publicidad, se podría argumentar que las personas más jóvenes tienen más tiempo discrecional que las personas que trabajan y es más probable que lleguen al cine antes de que comience la publicidad, por ende, se espera que las personas de mayor edad tengan una mayor exposición a la publicidad que los jóvenes.

La hipótesis $\mathrm{H} 3$ que relaciona el género con el nivel de recuperación de marcas se fundamenta en el hallazgo obtenido por Dunnett y Hoek (1996), que encontró mejores resultados de las mujeres en las pruebas de recuperación de anuncios de cine que los hombres. Por otra parte, el estudio de Dubow (1999) en Estados Unidos, sobre el recuerdo de publicidad en general, descubrió que los adultos más jóvenes recuerdan más anuncios que los adultos mayores, hallazgo que fundamenta la hipótesis H4. Finalmente, la idea que sugiere, que las percepciones de los consumidores sobre los anuncios publicitarios en los cines están relacionadas con su nivel de recuperación de marcas, está basado en los hallazgos de Stanley (1995) y Dill (1999) que muestran una aversión a la publicidad en una parte importante de la audiencia.

Para evaluar las hipótesis H1 y H2 se aplicará la prueba de chi-cuadrado, que es una prueba no paramétrica, debido a que las relaciones enunciadas solo involucran variables categóricas. Para las hipótesis H3 y H4 se utilizará la prueba t-Student, 
dada la naturaleza discreta del nivel de recuperación de marcas y la característica dicotómica de las variables demográficas. La hipótesis $\mathrm{H} 5$, involucra una variable discreta (nivel de recuperación de marcas) con una variable cualitativa de tipo politómica (percepción sobre la pertinencia de la publicidad), consecuentemente se correrá un modelo ANOVA para evaluar la relación entre ellas. Todas las estimaciones serán evaluadas bajo un nivel de significancia del $5 \%$.

\section{RESULTADOS}

\section{Contexto del estudio}

De las 195 personas entrevistadas en este estudio; 62 hombres y 99 mujeres tienen edades entre los 18 y 35 años, mientras, 29 hombres y 5 mujeres presentaron edades superiores a los 36 años. Lo que denota una mayor participación de los jóvenes y mujeres en la muestra extraída. A continuación, se muestra un resumen de los datos más representativos de las variables evaluadas sobre los Criterios de preferencias.

Tabla 3

Resumen de los Gustos y preferencia de los participantes

\section{Gustos y preferencias \\ Dato representativo}
Frecuencia de visita
El $33 \%$ asiste entre 2 y 3 veces por mes
Motivo de preferencia
El $30 \%$ considera a la variedad de horarios como la principal
Método de compra
El $45 \%$ compra sus boletos en las ventanillas
Género de película preferida
El $15 \%$ prefiere ver películas de terror
Actividad alternativa
El $37 \%$ elige ver Netflix

\section{Fuente y elaboración: Autor}

En promedio, las opciones preferidas de los entrevistados entre las distintas variables no superan el 32\% de participación. Lo que revela una distribución bastante uniforme entre las alternativas. Ciertamente, las preferencias de los Con respecto a las medidas de efectividad; el $77 \%$ de los entrevistados recuerdan haber ingresado antes de los anuncios publicitarios, el 14\% durante los anuncios y apenas un 9\% indicaron haber ingresado al terminar los anuncios, reflejando un nivel de exposición bastante representativo. En promedio, los entrevistados al terminar la función recuerdan haber observado anuncios publicitarios de al menos 2 marcas. Al mismo tiempo, un $36 \%$ consumidores en las decisiones que rodean su asistencia al cine son bastante heterogéneas, lo que dificulta las estrategias de captación de clientes en la industria del cine.

de los entrevistados considera que los anuncios publicitarios son atractivos, mientras que, a la mayoría que alcanza el $57 \%$ les resulta indiferente, y apenas un $7 \%$ lo considera molestoso. Evidenciado una percepción al menos neutral respecto al grado de aceptación de los consumidores sobre los anuncios publicitarios proyectados antes de cada película. 


\section{Exposición del público a la publicidad cinematográfica}

Una prueba de ji cuadrado $(\chi 2=7.493, \mathrm{p}=0.024)$ confirmó la no existencia de una relación significativa entre el género y el nivel de exposición de la publicidad. H1, que postula que existe una asociación entre el género y el nivel de exposición de la publicidad, no está respaldado por los datos. Al contrario, la edad si está asociada significativamente con la exposición publicitaria $(\chi$ $2=22.08, p=0.000$ ), por ende, se admite H2. En general, existe evidencia estadística para afirmar que los jóvenes entrevistados tienen más probabilidades de ingresar al cine antes de la proyección de los anuncios.

Tabla 4.

Exposición a la publicidad y variables demográficas.

\begin{tabular}{lcc}
\hline \multicolumn{1}{c}{ Variable } & Chi2 & Valor $\mathrm{p}$ \\
\hline Género & 0.7788 & 0.677 \\
Grupos Edad & 22.88 & 0.000 \\
\hline
\end{tabular}

Fuente y elaboración: Autor

\section{Nivel de recuperación de marcas}

$\mathrm{Al}$ aplicar la prueba $\mathrm{t}$ de muestra independiente, para comparar las puntuaciones medias del nivel de recuperación para hombres y mujeres. Se encontró una diferencia significativa entre los puntajes de hombres $(\mathrm{M}=2.27, \mathrm{SD}=1.12)$ y mujeres $(\mathrm{M}=$ $3.07, \mathrm{SD}=1.13 ; \mathrm{t}(195)=-5.2523, \mathrm{p}=0.000)$. Además, hubo una diferencia significativa entre los puntajes de adultos más jóvenes $(\mathrm{M}=2.64, \mathrm{SD}=$ 1.12) y adultos mayores $(\mathrm{M}=2.56, \mathrm{SD}=1.05 ; \mathrm{t}$ $(195)=2.3808, \mathrm{p}=0.018)$. Por lo tanto, las hipótesis H3 y H4 son compatibles. La evidencia sugiere que las mujeres y los adultos jóvenes tuvieron un mayor nivel de recuperación de marcas en los cines, que los hombres y los adultos mayores.

Tabla 5.

Recuperación de marcas y variables demográficas. Variable Estadístico $\mathrm{t}$ Valor $\mathrm{p}$

Género $-5.2523$ 0.000

Grupos Edad

2.3808 0.018

\section{Percepciones sobre la pertinencia de la publicidad en los cines}

Por último, los resultados del modelo ANOVA evidenció, que la interacción entre el nivel de recuperación de marcas y la percepción sobre la pertinencia de la publicidad no fueron
Fuente y elaboración: Autor

significativas $(p=0.445)$. Se concluye que las diferencias en las medias entre grupos de percepciones son iguales. Es decir, el nivel de recuperación de marcas no se ve influenciado por la percepción del consumidor sobre la pertinencia de la publicidad en los cines, la diferencias en los promedios de recuperación de marcas se debe 
netamente al azar o a la influencia de otro factor no explorado en esta investigación. Por lo tanto, H6 no está respaldada por los datos.

Tabla 6.

Resultados del modelo ANOVA sobre el nivel de recuperación de marcas frente a grupos con diferentes percepciones sobre la pertinencia de la publicidad en los cines.

\section{Variable dependiente}

\section{Nivel de recuperación de marcas - Puntajes promedio}

\begin{tabular}{lcccc}
\cline { 2 - 4 } & Atractiva & Indiferente & Molestosa & P - Value \\
\hline $\begin{array}{l}\text { Percepción sobre la pertinencia de la publicidad } \\
\text { en los cines }\end{array}$ & $2.63(1.13)$ & $2.58(1.09)$ & $2.60(1.21)$ & 0.445 \\
\hline
\end{tabular}

Fuente y elaboración: Autor

\section{CONCLUSIONES}

Como conclusión se puede identificar que el cine es un entretenimiento que no se ve afectado por los aumentos de precio que puedan tener, es decir el consumidor está consciente de lo que va a gastar, sin embargo, no deja de asistir, reconoce los precios elevados, quisiera que los disminuyan, pero sin embargo si estos no se modifican consume de la misma manera.

Las variables al momento de escoger el lugar en donde se disfrutará siempre serán como primordial la cercanía, lo que pone en ventaja a Supercines ya que cuenta con mayores puntos a nivel nacional, el segundo es la disponibilidad de horarios y formatos que este pueda ofrecer, la audiencia de los cines tiene un perfil colectivo, es decir no existe una cultura de asistir solo al cine, la mayoría de los encuestados reflejaron que disfrutan la compañía de su pareja, amigos o familia, y de esto dependerá el ticket de consumo, aunque en ocasiones se compren presentaciones grandes y en combo para compartir.

Es importante mencionar que estas marcas se han desarrollado a la par con el mercado ofreciendo plataformas virtuales para ser más eficientes al momento de la compra y reserva de entradas, lo cual está siendo aprovechado en su mayoría por usuarios de Samborondón.

De la misma manera en lo que se refiere a publicidad en los cines, este a pesar de ser un ingreso directo para los cines, para la audiencia en base a los resultados le resulta indiferente, para ellos puede ser indirecto, ya que la misma audiencia que mencionó que más recordaba cierta marca de hamburguesas, admitió ingresar este producto de manera oculta para poder disfrutarla al momento del rodaje de la película.

En base a estos criterios se puede decir que la publicidad en el cine será eficiente en base al tiempo e impacto que genere, ya que siempre llegará al usuario de manera indirecta, se considera que lo que a comida se refiere podría tener mucho más éxito, ya que, al visualizar el comercial, salir del cine, disfrutaran o tendrán apetito de la comida publicitada, y se verá ahí si realmente es o no impactante para la audiencia.

Finalmente, el mercado del cine tanto a nivel internacional como nacional es aquel que sigue creciendo en cuanto a innovación a la misma vez que los gustos del consumidor. Es por eso que marcas que buscan estar en los momentos de 
distracción del consumidor deberán aprovechar estos pequeños espacios publicitarios, tal vez no con un fin de generación de ventas instantáneas más sin embargo según el estudio este si genera recordación.

\section{REFERENCIAS}

Asociación Ecuatoriana de Agencias de Publicidad. (2014). Historia de la Asociación Ecuatoriana de Agencias de Publicidad. Recuperado de: <http://aeapecuador.com/historia.php>

La publicidad en la gran pantalla genera mayor impacto visual y mayor recuerdo. (3 de julio de 2008). Obtenido de PuroMarketing: https://www.puromarketing.com/23/4735/publicid ad-gran-pantalla-genera-mayor-impacto-visualmayor-recuerdo.html

ABC color. (12 de Junio de 2009). Obtenido de http://www.abc.com.py/articulos/funciones-de-lapublicidad-1181602.html

¿Qué es Cine? (1 de julio de 2015). Obtenido de Significado de Cine: https://www.significados.com/cine/

La Publicidad. (2019). Obtenido de Cyber Click: https://www.cyberclick.es/publicidad

Abelaez, C. C. (16 de Julio de 2018). Semana. Obtenido de https://www.semana.com/contenidoseditoriales/bello-sus-letras-lo-dicen-

todo/articulo/la-importancia-del-cine-y-formaciondel-publico/575545

Agredo Benavides, T., \& Matos Montoya, C. (Febrero de 2017). Repositorio USTA. Obtenido de ASPECTOS RELACIONADOS CON LA PREFERENCIA Y LA EXPERIENCIADE CONSUMO EN SALAS DE CINE: https://repository.usta.edu.co/bitstream/handle/116 34/3036/Agredothal\%C3\%ADa2017.pdf?sequenc $\mathrm{e}=1 \&$ isAllowed $=\mathrm{y}$

Angueta, R. A. (2018). Repositorio UASB. Obtenido de Marketing digital estrategias en redes sociales:

http://repositorio.uasb.edu.ec/bitstream/10644/598

4/1/T2478-MC-Angueta-Marketing.pdf

Cinemark. (2019). Cinemark del Ecuador S.A. Obtenido de https://www.cinemark.com.ec/

Conocimientos, Marketing, Ventas. (4 de abril de 2012). MERCADO: CONCEPTO, TIPOS, ESTRATEGIAS, ATRACTIVO Y SEGMENTACIÓN. Obtenido de LUIS MIGUEL MANENE:

http://www.luismiguelmanene.com/2012/04/04/elmercado-concepto-tipos-estrategias-atractivo-ysegmentacion/

Correa, V. S. (1 de Abril de 2014). Merca 2.0. Obtenido de https://www.merca20.com/la-eficaciadel-cine-como-medio-publicitario/

Dill, M. (1999). Coming attractions. Adweek, 40(34), 20-21.

Domingo, R. (s.f.). Revista Blogs. Obtenido de https://revistasblogs.com/tutorialesonline/publicidad-marketing-online/publicidad-ii/

Dubow, J. (1999). Advertising recognition and recall by age - including teens. Journal of Advertising Research, 35(5).

Dunnett, J., \& Hoek, J. (1996). An evaluation of cinema advertising effectiveness. Marketing Bulletin, 58-66.

Escárcega, A. (21 de junio de 2012). Historia del cine en México. Obtenido de Reconoce $\mathrm{Mx}$ : http://www.reconoce.mx/historia-del-cine-enmexico-primera-parte/

Ewing, M., Du Plessis, E., \& Foster, C. (2001). Cinema advertising re-considered. Journal of Advertising Research, 41, 78-85.

El Comercio. La rentabilidad de las películas ecuatorianas está a prueba. 20/11/2013. en Redacción de entretenimiento. (2013). Recuperado de: http://cinerama.ec/2013/01/17/el-comercio- 
revela-los-problemas-de-rentabilidad-de-laspeliculas-ecuatorianas/

Garces Albuja, G. (Octubre de 2012). Repositorio PUCE. Obtenido de PLAN DE NEGOCIO PARA LA CREACIÓN DE UN AUTOCINEMA: http://repositorio.puce.edu.ec/bitstream/handle/220 00/5141/T-PUCE-

5368.pdf? sequence $=1 \&$ is Allowed $=y$

García Velásquez, M.E. (2015). "Estudio del comportamiento del mercado cinematográfico ecuatoriano en el año 2012 y de la injerencia de los planes de negocios para la generación de ganancias". Fotocinema. Revista científica de cine y fotografía, 11, pp. 347-365. Disponible:_http://www.revistafotocinema.com/

Garcia, M.E. (2017). Relación entre el perfil del espectador de cine ecuatoriano y el grado de aceptación de las producciones nacionales estrenadas en el período 2012-2013. FUERA DE CAMPO REVISTA DE CINE Volumen 1, Número 4

Goyeneche-Gómez, E. (13 de Septiembre de 2012). Universidad de La Sabana. Obtenido de http://palabraclave.unisabana.edu.co/index.php/pal abraclave/article/view/2594/2893

Hidalgo, Á. E. (20 de septiembre de 2015). Cine y moral a inicios del siglo XX. El Telégrafo .

Inec. (2019). INEC. Obtenido de Instituto Nacional de Estadisticas y Censos: http://www.ecuadorencifras.gob.ec/estadisticas/

Martín, M. G. (septiembre de 2015). Comportamiento del consumidor de cine en salas: factores motivacionales y tipología del consumidor. Obtenido de Universidad Complutense de Madrid : http://eprints.ucm.es/46080/1/T39110.pdf

Mendiz, A. (s.f.). Obtenido de https://www.cinemanet.info/2008/11/la-influenciadel-cine-en-jovenes-y-adolescentes-completo/
Meneses, T., \& Geilany, M. (13 de Enero de 2017). Repositorio UCSG. Obtenido de Facultad de especialidades empresariales: http://repositorio.ucsg.edu.ec/bitstream/3317/7841 /3/T-UCSG-PRE-ESP-CIM-278.pdf

Muñoz, (A. 2015). Publicidad "made in Ecuador" y otras restricciones a la expresión publicitaria a partir de la entrada en vigencia de la Ley Orgánica de Comunicación. Universidad San Francisco de Quito.Vol 2 - No. 1

Olivas, O. (24 de noviembre de 2014). LA PUBLICIDAD EN EL CINE ES MÁS EFECTIVA QUE LA DE LA TV: ESTUDIO. Obtenido de Merca2.0: $\quad$ https://www.merca20.com/lapublicidad-en-el-cine-es-mas-efectiva-que-la-dela-tv-estudio/

Pérez Porto, J., \& Merino, M. (2018). Definición. Obtenido de Cine: https://definicion.de/cine/

Sánchez, Gsalán, M.B. (2010). La publicidad y la imagen en movimiento: primeros pasos del cine publicitario en España.Revista Pensar la Publicidad.Vol. IV, $\mathrm{n}^{\circ}$ 1, 79-96 Singh, S., Rothschild, M., \& Churchill, G. (1988). Recognition versus recall as measures of television commercial forgetting. Journal of Marketing Research, 72-80.

Stanley, T. (1995). Screens test co-marketing. Brandweek, 36(26), 26-31.

Supercines. (2019). Obtenido de Supercines: https://www.supercines.com/cartelera/guayaquil/d orado/236

Supercines. (2019). Supercines. Obtenido de https://www.supercines.com/

Veintimilla, A. (2018). El País se encamina a la exportación de publicidad. Obtenido de El Comercio:

https://www.elcomercio.com/actualidad/pais-seencamina-a-exportacion.html 
Wells, W., Burnett, J., \& Moriarty, S. (1995). Advertising, Principles and Practice (Tercera ed.). New Jersey: Prentice-Hall, Inc.

Wilson Astudillo Alarcón, C. M. (14 de Marzo de 2007). Universidad de Salamanca. Obtenido de http://revistamedicinacine.usal.es/es/volumenes/80 -vol4/num319/170-el-cine-como-instrumentopara-una-mejor-comprension-humana

Yuan, S. (2017). An investigation of the influence of cinema environment on advertising effectiveness. International Journal of Advertising . 